\title{
A Cloud-Enabled and Service-Oriented Framework for Context-Awareness towards Smarter Environments
}

\author{
S.Rajaraajeswari \\ Department of MCA \\ Raja Rajeswari College of \\ Engineering, Bangalore-560074
}

\author{
R. Selvarani, Ph.D \\ Dean and Professor \\ ACS College of Engineering \\ Bangalore-560074
}

\author{
Pethuru Raj Ph.D \\ Infrastructure Architect \\ IBM Global Cloud Center of \\ Excellence, Bangalore-560045
}

\begin{abstract}
Without any room for doubt, the journey towards the envisioned smarter planet is on the right track. Several things are happening in parallel to make the chosen route streamlined and smooth. Pragmatic ideas and concepts are being unearthed, scores of state-of-the-art technologies are fast emerging and evolving, processes are being greatly synchronized to be ready for efficiently tackling nextgeneration requirements, recurring situations and scenarios are being continuously captured, refined and deposited as widely usable and reusable patterns, policies and knowledge bases are being formulated towards the much-touted IT autonomy, insightfully integrated platforms are being designed, modular architectures are given extra thrust, infrastructures are being continuously optimized through a bevy of rationalization, virtualization, containerization, automation, and sharing techniques, natural and adaptive interfaces are being developed, facilitating frameworks and enabling tools, key guidelines, best practices, and pathbreaking products are being produced and made available across, etc. The idea is to empower IT to be multi-faceted, converged, dynamic, and smart.
\end{abstract}

In this paper, having understood the inherent need for contextawareness in order to develop and sustain a variety of smarter environments, we have described how we designed and implemented a viable and value-adding context-awareness framework and deployed it in a cloud environment.

\section{Keywords}

Service-Oriented Framework, Context Awareness, Smart Home, Cloud Computing

\section{INTRODUCTION}

\subsection{The Journey towards Smarter Environments}

These days, creating and sustaining smarter environments (say smarter homes, hotels, offices, cities, buildings, governments, energy, utility, etc.), [1] have been gaining immense momentum with the faster emergence and evolution of deeper and extreme connectivity, service-enablement, cloud computing, data analytics, mobility and social technologies. There are a number of powerful automation and integration platforms, patterns, processes, and practices signaling the onward journey towards the building and sustenance of active and adaptive environments [2-3]. For an example, homes across the globe are being continuously stuffed, saturated and stunned with a variety of exquisite automation elements in order to sharply increase the quality of human living, to enhance human productivity and to guarantee heightened care, comfort, convenience and choice. However, unfortunately devices, which come in different shapes, scopes and sizes, from multiple vendors leveraging different standards and technologies, are obstructing the free flow of information within themselves, in locating one another dynamically in order to communicate, compose and collaborate to be highly beneficial for home owners and occupants with diverse needs. That is, device heterogeneity and multiplicity lead to the unwanted IT complexity. Hence the moot and major question in everyone's lips is how to displace and delegate the growing complexity.

The tremendous acceptance and adoption of the raging cloud idea has come out as a blessing in disguise for decimating some of the critical and crucial IT complexities. As indicated above, currently the most vocal method for arriving at smarter places is the prominent centralized and box-based approach. It is however found that this overwhelming approach ultimately would lead to more complexity putting barriers in accommodating unpredictable changes and furthering innovations. This has in turn propelled professionals and pundits to ponder about scores of viable and veritable alternatives. The widely discoursed and discussed cloud technology is being found to be an extremely good fit for saying goodbye to the perpetual problems and for welcoming smarter environments.

The core principles behind smarter environments are

Displacing and delegating the complexity

Accommodating unforeseen changes

Facilitating innovation such as open business model

Enhancing consumability through intuitive interfaces

In this paper, we have submitted all the details regarding the context-awareness framework, the key components and algorithms, and how the framework is plentifully empowered through the cloud integration. This cloud-enabled framework is very extensible in order to absorb any kinds of improvisations in the cloud technology, device integration, etc. in the days to unfold.

\subsection{The Design Principles of the Context- Aware Framework [4]}

IT product vendors and academic institutions have been working together and bringing forth a number of nimbler computing paradigms in order to accomplish widely changing expectations of businesses and users. However as it turned out, the reality is that computing systems could do only preprogrammed things. That is, software applications on receiving specific inputs are capable of producing 
predetermined outputs. That is, inputs are typically fixed beforehand and the outputs are as per the expectations.

Users' context is being primarily decided based on the implicit capability of establishing seamless and spontaneous connectivity and integration with a dynamic pool of software services and applications (social, enterprise, mobile, embedded, and cloud), multiple data sources, scores of devices, appliances and sensors in the user(s)' environment. In a nutshell, the fast-emerging paradigm of context-aware computing is to produce context-sensitive outputs for human beings to act upon that with all the clarity and confidence.

\subsection{A Sample Use Case}

As mentioned above, there are several types of smarter environments emerging and attractive use cases are being proposed. Ambient Assisted Living (AAL) is a very prominent use case for smarter homes. The need is to empower bed-ridden, debilitated, and diseased people to lead an independent and digitally assisted living. For example, if a person living alone wants to have a cup of filtered coffee, he or she can instruct the coffee maker in the kitchen through a smart phone command. Once the coffee is ready, a robot can be ordered to fetch the prepared coffee to his / her bedside. Similarly medicine cabinets can have an alert / reminder facility to take tablets in time, the movement of the person can be monitored remotely, household items can be bought over through mobile commerce sites, food stuff can be purchased online, all kinds of consumer electronics, energy managers, kitchen vessels and wares, instruments, micro ovens, infotainment and edutainment systems, dish washers, toasters, refrigerators, and healthcare monitors, etc. can be instrumented and interconnected to have intelligent behaviors thereby the everyday activities and requirements can be met with all choice and convenience. New services getting loaded in cloud-based platforms and infrastructures can be automatically subscribed by home-bound devices to be relevant for home owners and occupants.

\section{LITRATURE SURVEY}

Ad hoc networking of sensors and actuators actually produce a large amount and variety of data, which need to be systematically collected, polished and processed to transition them to become usable knowledge. This processed data could inform the location of devices and people, the state of assets, users' preferences, time constraints, moods, the place of action, etc. These are generically called as context-awareness, which is very important for next-generation applications to be distinct in their operations, offerings and outputs. Vijay Borges [5] has explored different context information fusion techniques by comparing a large number of solutions, their methods, architectures and models.

There have been many game-changing works in the fields of device integration concepts, standards, frameworks, platforms, and tools. A wide variety of devices, applications, data sources, services, and sensors need to be integrated seamlessly to extract context-sensitive information. A survey about context posturing and reasoning out some techniques instead of research projects by Bettini et al [6]. A survey on context mobile networking has been carried out by Makris et al [7].

Sayed A.Quadri [8] has worked on multi-sensor data fusion to overcome the limitations of individual sensors and to produce accurate, robust and reliable estimates based on multisensory information. Data fusion algorithm plays significant role in achieving reasonable performance. In his paper, it is presented an algorithm that is employed to fuse data obtained from accelerometer and gyroscope in an inertial measurement unit (IMU).

There are other researchers working on different aspects of context-aware computing, which is the core and central concept for establishing and sustaining next-generation smarter environments that could understand and provide peoples' needs in time in an unobtrusive manner.

\section{A FRAMEWORK FOR REAL-TIME CONTEXT-AWARENESS 3.1 The Principal Modules of the Context- Aware Framework}

There are sensing, perception, vision, decision-enabling, and actuation technologies flourishing to create and sustain context-aware environments. People get excited when they get all kinds of information, commercial transaction, knowledge and physical services based on their situation (location, time, etc.) and their various needs (mental, physical, social, etc.). There are cyber applications, cloud-based services, user devices, communication gateways and device middleware, digitalized and interconnected objects, etc. collectively contributing for the swift implementation and delivery of cognitive context-aware services to users. Figure 1 shows the reference architecture of the context aware framework. The framework for context-aware computing mainly possesses five modules.

\subsubsection{Data collection and cleansing}

This is a prime component for carefully collecting data from distributed and difference sources. With the continued growth of data generators and extractors (sensors and actuators, smart phones, social sites, enterprise as well as cloud data centers, devices and instruments from research labs, machines from manufacturing plants and floors, etc.), multi-formatted and faceted data are being gathered these days. Further on, data gleaned need to be subjected to a series of tasks such as transforming and polishing in order to make them compatible for the target data storage environment. With the emergence of new kinds of data base management systems (SQL, NewSQL and NoSQL), the choice of data management is very critical for the framework. There are data connectors, drivers, adapters and integrators made available by product vendors to simplify this arduous task. Data virtualization is a new term in the industry gaining momentum for data collection and synchronization in highly distributed and disparate environment.

\subsubsection{Data storage}

Once data get refined is duly stored in an easy-to-access and use storage infrastructure such as a database (DB), data cube and mart or data warehouse (DW). This is for enabling users to query the data warehouse to retrieve right and relevant information to plan ahead and proceed with clarity and confidence. This is a kind of pull mechanism and is a batch processing. Slicing, dicing, report generation and other kinds of macro as well as micro-level operations can be accomplished on data stored to squeeze out usable information. 


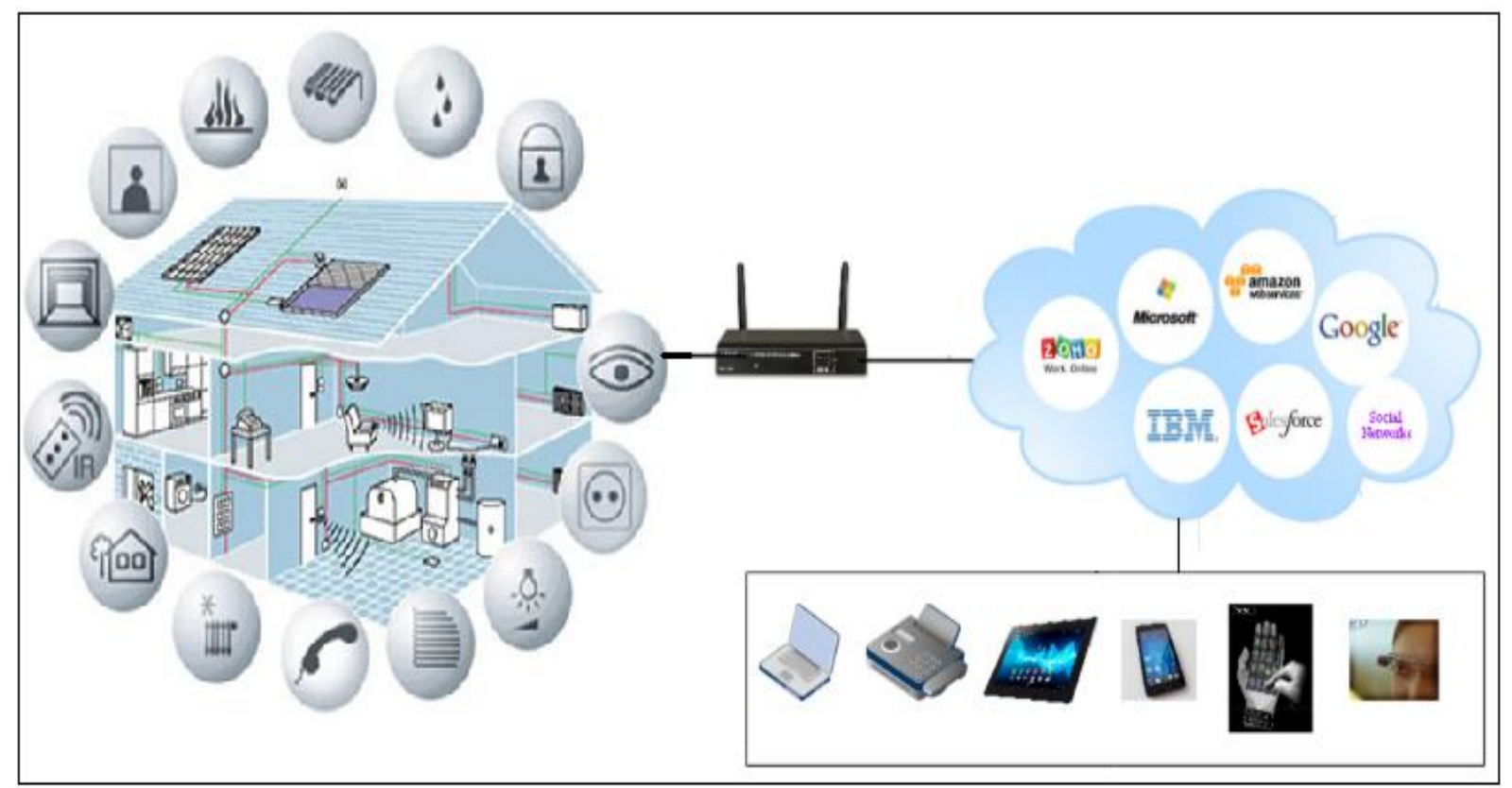

Figure 1: The reference architecture for the context aware framework

\subsubsection{Data interpretation}

On the other hand, as data pours in different places, contextsensitive information needs to be retrieved and dispatched to the concerned people through a variety of devices, displays, etc., centralized monitoring, measurement and control systems, any appropriate actuation systems, etc. That is, a competent real-time data interpretation mechanism has to be in place for extracting situation details quickly to act on them. Knowledge discovery is a crucial cog in context-aware computing. There is a need for having special knowledge bases, policies / rules repository, and other viable solutions to facilitate accurate interpretation of incoming data to extract timely insights. Thus knowledge engineering and enhancement are achieved through multiple tasks such as data integration, aggregation, classification, clustering, composition, processing, analyzing, mining, etc. done individually and collectively.

\subsubsection{Cloud enablement}

The context-aware framework has a module to enable the cloud connectivity. These days, sensors and devices at our everyday environments are not only connected with one another in the vicinity but also with remotely hosted applications, services and data in cloud environments. With the device integration standard such as OSGi, devices in our physical places are getting empowered by dynamically downloading all kinds of enabling services, installing and configuring them to be highly relevant for the widely discoursed and discussed digital living. Thus cloud integration is an indispensable for futuristic context-aware solutions. These days, different sorts of data are getting generated outside our living environments and they are of high value for people if leveraged smartly. For example, there are unprecedented advancements in the forms of social networking sites, knowledge communities, smarter cars with in-vehicle infotainment systems, financial industries pumping trillions of bytes every day, etc. The data growth being project is simply phenomenal.

\subsubsection{Data dissemination}

Knowledge engineered needs to be packaged and shared in a preferred and presentable format to users. Reports, maps, charts, and graphs are the main mechanisms for knowledge share. Data visualization is a popular topic bombarded with a bevy of techniques, tools and tips facilitating the dissemination of knowledge to people in time.

\subsection{The Context Engineering Infrastructure and Platforms}

There are new types of platforms and infrastructures emerging and evolving fast to enable IT to be radically tuned towards meeting the constantly changing requirements of end-users. Self-, surroundings and situation-awareness is being presented as an essential trait for the forthcoming smarter world. Accordingly worldwide IT vendors are working in harmony to bring forth competent products and platforms. There are appliances, expertly integrated systems, and specially engineered systems with in-database and in-memory processing capabilities. Software bundles are turning out to be a hugely successful phenomenon for simplifying and streamlining application deployment, performance tuning, infrastructure management, etc. There are service delivery platforms (SDPs) comprising service bus, integration engines, orchestration containers, etc. Device middleware is another zooming domain with growing mind and market shares. Realtime analytics, predictive analytics, and even prescriptive analytics are gaining momentum with the availability of sophisticated technologies and processes. Stream computing is a new compute paradigm contributing spectacularly for real-time knowledge discovery and supply. Data mining has been a powerful subject of study and research for data analytics.

\section{THE FRAMEWORK IMPLEMENTATION DETAILS}

We have implemented the framework and used the same in order substantiate the idea of context-awareness in an effective and efficient manner. The system configuration details are given below.

Quad-core Processor of 2.2 Ghz, 8 GB RAM Storage, 500 GB Disk Storage, Java 7 is installed and the environment variables are appropriately added. 
phpMyAdmin is a free software tool written in PHP, intended to handle the administration of MySQL over the Web. Java language is the primary language leveraged for the framework implementation. The values from different sensors deployed plentifully in a smarter home get updated every time in a database.

\section{THE ALOGORITHMS FOR DATA CAPTURE AND INTERPRETATION}

A set $S=\left\{s_{1}, s_{2}, s_{3}, \ldots \ldots \ldots s_{n}\right\}$ represents the sensors embedded in a home environment.

Different sets, each representing set of sensor data at a particular time gets automatically updated to a database. Hence $\mathrm{S}_{\mathrm{t} 1}, \mathrm{~S}_{\mathrm{t} 2}, \mathrm{~S}_{\mathrm{t} 3} \ldots \ldots \mathrm{S}_{\mathrm{tn}}$ are the data sets that got updated in a database at different time intervals.

$\{\mathrm{s} 1 \in \mathrm{St} 1 \mid \mathrm{s} 1$ recorded at $\mathrm{t} 1\} ; \mathrm{St} 1 \neq \varnothing$ Also \# St1 =n $\mid \mathrm{n}>0$ [Representing cardinality]

$\{\mathrm{s} 2 \in \mathrm{St} 2 \mid \mathrm{s} 2$ recorded at $\mathrm{t} 2\}$

$\{\operatorname{sn} \in \operatorname{Stn} \mid$ sn recorded at tn $\}$

\subsection{Algorithm Involved in Categorization}

$\mathrm{X} \& \mathrm{Y}$ are the initial sets with

IP: $X=\left\{s_{1}, s_{2}, \ldots . . s_{n}\right\} \& Y=\varnothing$

OP: Categorized sets.

Start:

$\mathrm{X}=\{\mathrm{x} \in \mathrm{s} \mid \mathrm{S}(\mathrm{x})\}$

While (not the end of set)

if $(x \mid x>$ threshold $)$

$$
Y=\{x \in X \mid S(x)\}
$$$$
\text { else }
$$

No changes to the set end loop;

end;

\subsection{Integration Function}

From the set $\mathrm{S}$ into the set $\mathrm{P}$, the set of functions is the set $\mathrm{S} \rightarrow \mathrm{P}$.

The signature as an operator on sets, under the interpretation of $(\rightarrow)$ :

$f: S \rightarrow P$ is a member of the set $S \rightarrow P \mid f \in S \rightarrow P$

Defining the function as:

$\mathrm{f}(\mathrm{x})=(\mathrm{x} * 100) /$ Threshold $; \quad \mathrm{x} \in \mathrm{S}$

\subsection{Analyzing User's Request}

$\mathrm{S} 1 \& \mathrm{~S} 2 \mid \mathrm{S} 1 \subset \mathrm{S} \& \& \mathrm{~S} 2 \subset \mathrm{S}$

$\mathrm{D}=\mathrm{S} 1 \times \mathrm{S} 2=\{(\mathrm{x}, \mathrm{y}): \mathrm{x} \in \mathrm{S} 1$ and $\mathrm{y} \in \mathrm{S} 2\}$.

E.g.: If $S 1=\{$ Temp_sensor, Smoke_sensor $\} \& S 2=$

\{Fire sensor\}

Then, $\mathrm{D}=\mathrm{S} 1 \times \mathrm{S} 2=\{($ Temp_sensor, Fire_sensor $)$, (Smoke_sensor, Fire_sensor) $\}$.

$\Rightarrow$ To analyze user query "Possibility of fire"... The elements in the set D are used.

$\Rightarrow$ If both the Temp_sensor \& Fire_sensor crosses the threshold, then fire accident may occur in the house.

\section{THE FINITE STATE AUTOMATA CONCEPT FOR CONTEXT AWARENESS}

As we all know, the concept of finite state automata is being widely recognized as the key mechanism for unambiguously representing even highly complex behavior of mission-critical systems. Being a leader in optimally modeling various actions and reactions of business as well as IT applications, we strongly feel that the finite state machine (FSM) is more appropriate for highly dynamic smarter environments. The widely leveraged FSM models are not only facilitating the representation but also enable seamless transmitting and persisting FSM models. Here comes the formal definition of the FSM concept.

A Non-Deterministic finite state automaton or acceptor nondeterministic finite state machine is quintuple $\left(\sum, \mathrm{S}, \mathrm{Q}, \mathrm{q}_{0}, \delta, \mathrm{F}, \mathrm{X}, \mathrm{K}\right)$ in which $\sum$ is the input alphabet taken from non-empty finite set of symbols $\left\{\lambda_{1}, \lambda_{2}, \ldots \ldots \ldots \ldots\right.$, $\left.\lambda_{16}\right\} \cup\left\{L_{0}, L_{1}, L_{2}, \ldots \ldots, L_{7}\right\}, S$ is the switching element with $s=\{0,1\}$ indicating the two states whether it is switched(1) or not $(0), Q=\left\{\mathrm{q}_{0}, \mathrm{q}_{1}, \ldots \ldots \ldots \ldots, \mathrm{q}_{18}\right\}-\left\{\mathrm{q}_{15}\right\} \mathrm{U}\{\mathrm{L}, \mathrm{M}, \mathrm{H}\}$ is a nonempty set of finite states with the state space $\mathrm{R}^{\mathrm{n}}$ and with $\mathrm{q}_{0}$ as the initial state, $\delta$ is the state transition function which is defined as $\delta: \mathrm{Q} \times \sum \rightarrow \mathrm{p}(\mathrm{q})$ that returns set of states, $\mathrm{F} \subseteq \mathrm{Q}$ with set of final states from the $\operatorname{set}\left\{\mathrm{q}_{3}, \mathrm{q}_{8}, \mathrm{q}_{12}\right\} \cup\{\mathrm{L}, \mathrm{M}, \mathrm{H}\}, \mathrm{X}$ is the set of exceptions $\left\{E_{1}, E_{2}, E_{3}, \ldots \ldots \ldots \ldots . . . . . . E_{7}\right\}$ and finally with set of key constraints represented by $\left\{\mathrm{C}_{1}, \mathrm{C}_{2}, \ldots \ldots, \mathrm{C}_{5}\right\}$.

- Now, $\langle\mathrm{Q}, \mathrm{E}\rangle$ is a directed, controlled context aware automated graph and $\mathrm{R}^{\mathrm{n}}$ is the automaton's state space. We can have a stub with its definition as follows.

- Hop: $\mathrm{R}^{\mathrm{n}} \rightleftharpoons \mathrm{R}^{\mathrm{n}}$ showing that the state changes when traversing along edge $\mathrm{e} \subseteq \mathrm{E}$ with $\left(\mathrm{q}_{0} ; \mathrm{x}_{0}\right) \in \mathrm{Q} \times \mathrm{R}^{\mathrm{n}}$ as inceptive situation.

- The transition relation: $(\mathrm{q} ; \mathrm{x}) \rightarrow\left(\mathrm{q}^{1} ; \mathrm{x}^{1}\right)$ is possible if and only if the state of entity is in the domain of hop/ jmp.

\subsection{A Sample Use Case}

There are growing arrays of smart environment applications. Especially with the voluminous availability of devices for smart homes, hospitals and hotels, there is an eruption of people-centric use cases. The operations of a galaxy of devices, machines and other electronics within an environment are grandly automated and people-aware processes are fully automated with device interactions and orchestrations. The table $1 \& 2$, and figure 2 below vividly illustrates an usage.

\section{THE FRAMEWORK ADVANTAGES}

This framework has intrinsically used a bevy of proven algorithms for various steps ranging from data collection to knowledge engineering. This is a web-based application so that anyone with the Internet connectivity can use this system for making his environment smart. This is being polished further in order to work in smartphones (Android, iOS, BlackBerry, and Windows Phone). Not only in mobile phones but also in all kinds of electronic devices (wearable, portables, fixed, etc.). With the latest phones are being stuffed with a variety of sensors, context-awareness can be being speeded up 
and simplified through this framework application. That is, all the environmental sensors are in direct interactions with sensors attached with phones to enable them to act as the most prevalent and popular gateway for context-awareness for people in different locations in their daily lives.

\section{THE PERFORMANCE EVALUATION}

Table-I Different Transitions of action

\begin{tabular}{|l|l|l|l|}
\hline Symbol & Transitions & Symbol & Transitions \\
\hline$\lambda 1$ & $\begin{array}{l}\text { Switching on } \\
\text { lights in balcony }\end{array}$ & $\lambda 9$ & $\begin{array}{l}\text { Turning on } \\
\text { electric stove }\end{array}$ \\
\hline$\lambda 2$ & $\begin{array}{l}\text { Going to wash } \\
\text { room }\end{array}$ & $\lambda 10$ & $\begin{array}{l}\text { Null } \\
\text { transition }\end{array}$ \\
\hline$\lambda 3$ & $\begin{array}{l}\text { Going to } \\
\text { cooking room }\end{array}$ & $\lambda 11$ & $\begin{array}{l}\text { Turning on } \\
\text { AC in LR }\end{array}$ \\
\hline$\lambda 4$ & $\begin{array}{l}\text { Going to bed } \\
\text { room }\end{array}$ & $\lambda 12$ & $\begin{array}{l}\text { Checking } \\
\text { windows }\end{array}$ \\
\hline$\lambda 5$ & $\begin{array}{l}\text { Switching on } \\
\text { LR lights }\end{array}$ & $\lambda 13$ & $\begin{array}{l}\text { Switching on } \\
\text { exhaust fan }\end{array}$ \\
\hline$\lambda 6$ & $\begin{array}{l}\text { Switching on } \\
\text { wash room } \\
\text { lights }\end{array}$ & $\lambda 14$ & $\begin{array}{l}\text { Switching on } \\
\text { bed room } \\
\text { lights }\end{array}$ \\
\hline$\lambda 7$ & $\begin{array}{l}\text { Turning on } \\
\text { cooking room } \\
\text { lights }\end{array}$ & $\lambda 15$ & $\begin{array}{l}\text { Opening } \\
\text { windows }\end{array}$ \\
\hline$\lambda 8$ & $\begin{array}{l}\text { Turning on } \\
\text { electric stove }\end{array}$ & $\lambda 16$ & $\begin{array}{l}\text { Closing } \\
\text { windows }\end{array}$ \\
\hline
\end{tabular}

The figure 3 below clearly illustrates the CPU usage of two sample context-aware applications: smart homes and contextaware hospital.

The performance evaluation of the traditional approach (TA) and the new approach introduced by us using the REST API + Automaton is indicated in figure 4 . The performance of our method is comparatively better.

Table-II Different states of actions

\begin{tabular}{|l|l|l|l|}
\hline Symbol & \multicolumn{1}{|c|}{ States } & Symbol & \multicolumn{1}{|c|}{ States } \\
\hline q 0 & Balcony & q 9 & Electric stove \\
\hline q 1 & $\begin{array}{l}\text { Lights switched } \\
\text { on, Staying in } \\
\text { LR?? }\end{array}$ & q 10 & Lying on bed?? \\
\hline q 2 & $\begin{array}{l}\text { In wash or } \\
\text { cooking or bed } \\
\text { room?? }\end{array}$ & q 11 & AC turned on \\
\hline q 3 & TV switched on & q 12 & $\begin{array}{l}\text { Exhaust fan } \\
\text { switched on }\end{array}$ \\
\hline q 4 & Wash room & q 13 & $\begin{array}{l}\text { Lights switched } \\
\text { off }\end{array}$ \\
\hline q 5 & $\begin{array}{l}\text { Cooking room } \\
\text { q 6 }\end{array}$ & $\begin{array}{l}\text { q 14 room } \\
\text { Lights switched } \\
\text { on }\end{array}$ \\
\hline q 7 & $\begin{array}{l}\text { Lights switched } \\
\text { on }\end{array}$ & q 16 & $\begin{array}{l}\text { Checking } \\
\text { window }\end{array}$ \\
\hline q 8 & $\begin{array}{l}\text { Lights switched } \\
\text { on }\end{array}$ & q 18 & Window opened \\
\hline
\end{tabular}

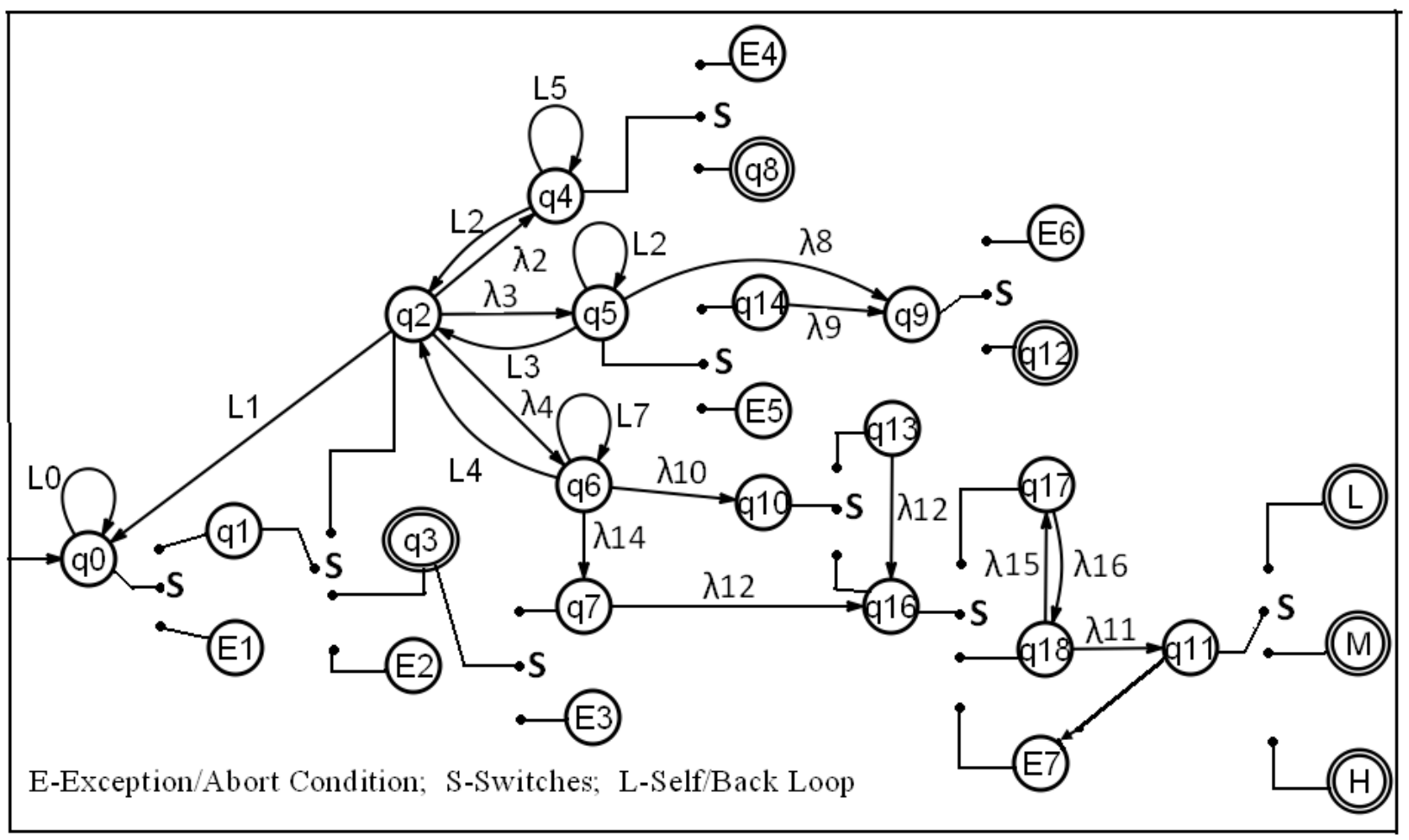

Figure 2: Smart homes - The finite automata representation 


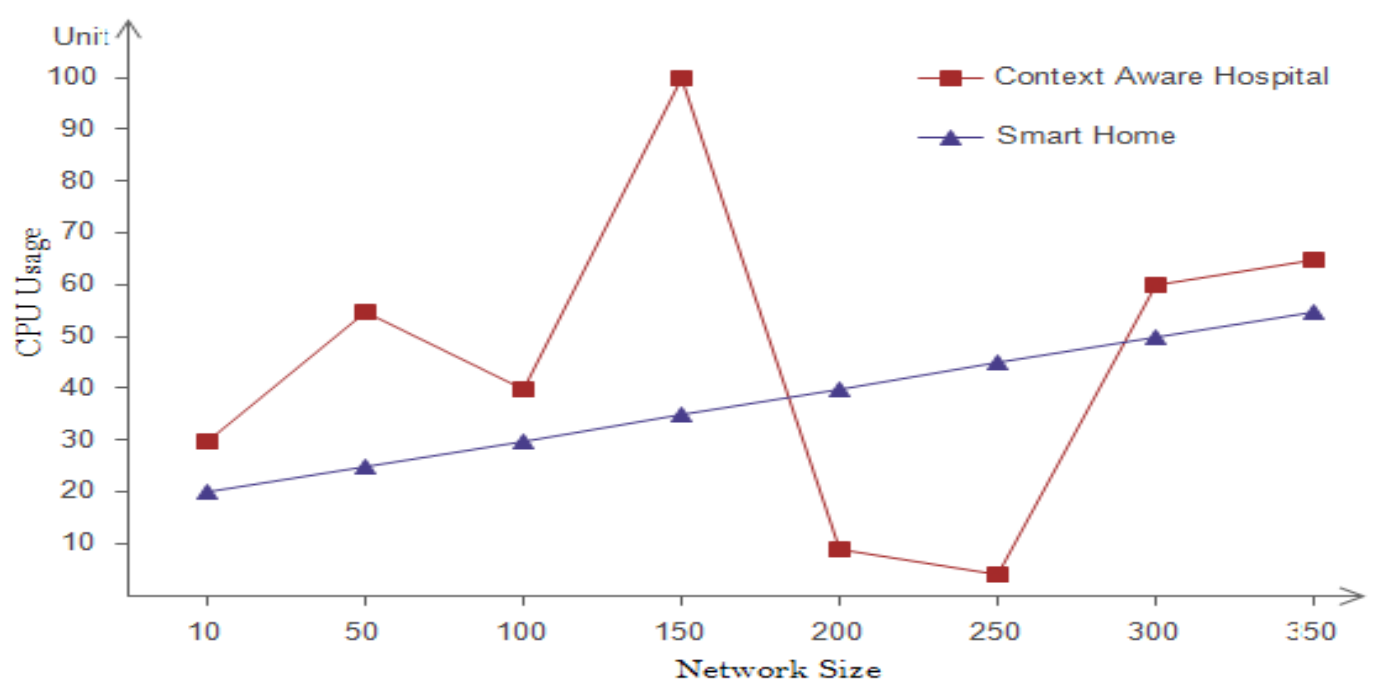

Figure 3: CPU usage of smart homes and context-aware hospital

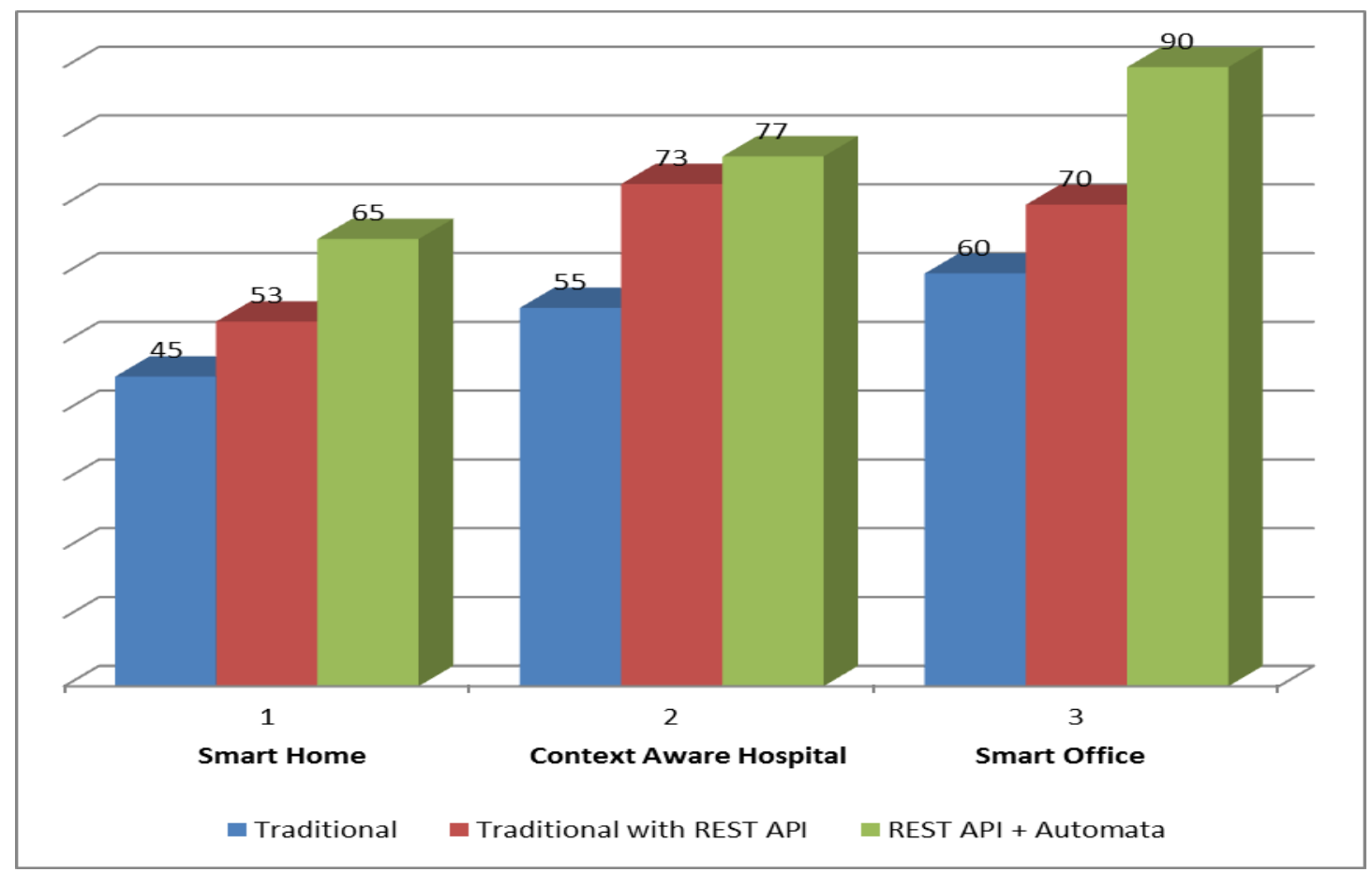

Figure 4: Performance evaluation of the traditional approach versus REST API + Automaton approach in context-aware hospital

The thorough analysis is made among the methods that implement context-aware computing. This is presented in the bar graph where comparison is depicted among the traditional approach (TA), the TA with REST API and the third approach (TA with REST API and Automata). The performance shown is better for different environments (Smart home, smart office, and Context-aware hospital).

\section{THE NEED FOR THE CLOUD ENABLEMENT}

Infrastructure optimization is the gist of cloud computing. It is all about transforming traditional data centers and server farms to become virtualized, automated, and shared environments so that moving on-premise compute resources (applications, platforms, and hardware resources such as compute, memory, storage, network etc.) to cloud centers makes a strategic business decision. Cloud environments are agile, optimized, and software-defined through smartly applying a host of technological solutions.

Cloud host online and on-demand applications that can be accessed remotely. This is analogous to centralized and federated electricity generation utilities by government or private people and people can avail the required electricity for a small fee for the amount or duration of usage. In the similar way, the total compute facility as a collaborative pool of virtualized and automated virtual and baremetal servers is 
being put up in a central place to be actively monitored, managed, and maintained by those who are having the meticulous education, experience and expertise. And any subscriber can subscribe for the relevant and right amount of compute / storage / network needs at any point of time. That is, it is all about utility-like resources that support plug and play paradigm inherently. All sorts of infrastructural and usage complexities are displaced and delegated into experienced hands. The resounding success is not only due to the extra abstraction obtained through this cloud paradigm but also even common people with an Internet connectivity can easily find, and access the growing list of cloud services, applications, data, and other resources immediately.

In a single stroke, the much-discoursed and deliberated cloud paradigm brings to the table an altogether different business, pricing, delivery and usage model, which is significantly deviating from the conventional model. Institutions, individuals, innovators, etc. can make use of cloud resources in innumerable ways. All the right and relevant ICT infrastructures are being cloud-enabled to be subscribed and used by anyone anywhere anytime any device. Increasingly IT development, deployment, and enhancement tasks are being slowly yet steadily shifted to large-scale cloud centers that are empowered with all kinds of software engineering frameworks, integrated development environments, testing suites, software containers, integration middleware, and delivery platforms. In a nutshell, cloud is the most soughtafter, hot, and happening place.

Services and data made available in the cloud can be more ubiquitously accessed, often at much lower cost. This in turn increases their value by creating opportunities for enhanced collaboration, integration, and analysis on a shared common platform. At its heart, cloud computing is a new operational and business model for IT application hosting.

\section{THE CONUNDRUM OF SERVICE PARADIGM}

Being infrastructure-independent, services can be easily transmitted over any network and deployed, configured, executed, monitored, and maintained on any standardscompliant platform, container and engine. Services are extremely flexible for spotlessly converging with other system building-blocks such as agents, aspects, formalized models, POJOs, events, composites etc. These remarkable characteristics have clearly elevated the value and power of SOA in quickly and easily modernizing languishing and sagging applications, integrating disparate and distributed systems, and realizing exciting and elegant composites. The near as well as the long-term implications are that the complicated, closed, and costly business IT is definitely destined for a major overhaul and turnaround into affordable, adaptive, and agile IT.

\section{SMART HOME ELEMENTS}

A growing variety of smart sensors, systems, services and solutions are set to decorate future homes. That is, homes are to be extremely technology-empowered and splurged. Home appliances, utensils, and gadgets are being empowered to talk with each other. That is, ordinary and everyday objects are being web-enabled and are empowered with relevant and right intelligence through the internal as well as external incorporation of additional electronic modules. All kinds of divisiveness are getting eliminated through a host of measures such as standardization, adaptors, bridges, middleware, common APIs, etc. Plug and play capabilities is being guaranteed. Both today as well as tomorrow's devices are manufactured and modernized to connect and collaborate with one another in realizing people-centric tasks.

\subsection{Smarter Home Capabilities}

The outcomes of smarter homes are many including making consumers' lives more productive, healthier and happier. The four service areas are

* Entertainment and Convenience - The richness of the Internet is now available through the TV. Now consumer electronics companies can create open platforms for new lines of flat panel televisions that feature a portal which personalizes entertainment content from numerous broadcasters and move studios

* Energy Management - Future demands on the electrical grid will encourage minute by minute home appliance management to priorities energy services while delivering automatic savings to owners. Automatically synchronizing lighting, home appliances, climate and environmental sensors and all household smart objects sharply minimizes energy consumption based on changing environment conditions and usage patterns in the home.

* Safety \& Security - Many insurers now offer discounts for existing centralized alarm services using sensors and IP surveillance cameras. The ability to deploy home sensors that can instantly notify the homeowner, selected neighbors, or the police and fire departments can enhance home security. These services can also empower family members to remotely check on the safety of children and the well-being of elders

* Health \& Wellness - Healthcare providers could continuously monitor their patients with implanted devices or other at-home medical devices without hospitalization. Smarter home scales and sensors monitor fitness, well-being, and advanced parameters consistently. These home health electronic devices can collect evaluative information about current health condition for disease management and prevention and guarantee overall wellness.

\subsection{Smarter Home Services in the Cloud}

Home automation elements are being manufactured in plenty, linking of distributed and disparate devices is being smoothened out, conceiving newer and nimbler services is in full swing, enabling frameworks, infrastructures are virtualized and pooled, and proven processes are in place, clustered, brokered, federated, and cloud architectures are being worked out etc.

The present-day smart home involves home servers into the home for integrating diverse household electronics and for their effective control and usage. However, the need to manage, even if done remotely, very complex IT devices in the home has prevented this approach from being widely adopted. A more effective approach is to connect the devices to services in the cloud. This enables the centralization of services. Putting the intelligence into services in the network cloud reduces the complexity of managing software in the home devices and simplifies the interoperability of devices. This is accomplished by bridging the services throughout the 
network through web services interfaces, translating the different device protocols to a common platform, then connecting the devices through the network cloud. This is a far less expensive and more flexible way to aggregate services and compose new services existing devices than past attempts. This takes out the complexity out of the connected devices in the home. The consumer moves from being the IT manager of their home to a consumer of services through their connected devices.

A local services "clone" or services cache in the home can safeguard the availability of services against network problems and guarantee continuous high quality, all with simple control by the consumer. With new cloud services, new possibilities exist for an appliance as simple as a pool pump or refrigerator when it has the ability to sense and respond to changing conditions, to communicate with other systems and to inform decision-making.

\subsection{How Cloud Enables Smarter Homes?}

Cloud technology creates an ideal environment for an intelligent, highly efficient and highly flexible utility approach to services in the network. At the hardware level, it enables the flexible, dynamic, and low-touch provisioning of resources to applications. The creation of virtual service images supports the easy lifecycle management and deployment of services. Finally, standardized web services interfaces to the services enable the dynamic composition of individual services into flexible solutions in a plug-and-play mode [9].

The cloud for managing the consumer services can extend directly into the home. A services' clone can directly interact with services in the network, in effect becoming part of the cloud. This clone can function as a limited and local replica of some services, delivering control even in the case of a network failure. It is also possible to ease the connection of home devices to the network by translating protocols and acting as traffic concentrator. The ability to adapt any type of network and application protocols increases the choice of devices and services for consumers.

Cloud technology brings agility, affordability and above all autonomy to IT operations thereby business automation, and augmentation are set to be realized fast [10]. It reduces the resources cost by enabling more efficient allocation of fractional hardware resources to virtual service images. The open platform environment permits devices to be connected, enabling interoperation and remote access of services. This will create an ecosystem based on industry standards, driven by industry leaders. Using SOA, services with different protocols and data types can be easily connected. The application services can also take advantage of common infrastructure services, such as CRM, billing, etc.

\section{CONCLUSION}

Context-awareness is an important ingredient for nextgeneration applications and environments in order to be distinct in their actions and reactions. There are a number of connectivity and integration technologies facilitating the realization of connected devices, smart sensors and their ad hoc networks, cyber physical systems (CPS), wireless machine-to-machine (M2M) communication, device-to-cloud
(D2C) integration, etc. All these directly and indirectly facilitate the extraction of context data towards the muchneeded context-awareness. That is, any software applications or even users empowered with context information can be of distinctively unique in their decisions, deeds and deals. For example, context-aware smartphones will be of much help in near future for mobile phone users. Context-aware applications and services will be of high use for the forthcoming knowledge era.

In this paper, we have brought forth an easy-to-use contextawareness framework leveraging the proven service paradigm, the cloud paradigm, finite state machine (FSM) concepts, and a couple of stabilized software platforms and languages. This framework is designed and developed in a modular fashion so that all kinds of futuristic changes can be easily incorporated to take on all kinds of emerging challenges. The framework has been subjected to a series of tests and the results are really positive. There are several other things to be added into this framework to make it more compact and comprehensive.

\section{REFERENCES}

[1] A white paper 2010. "The IBM Vision of Smarter Homes enabled by Cloud Technology". www.ibm.com

[2] Lopes, F., Delicato, F. C., Batista, T., Cavalcante, E., Pereira, T., Pires, P. F., Mendes, R. 2014. OpenCOPI: Middleware integration for ubiquitous computing. International Journal of Parallel, Emergent and Distributed Systems, 29(2), pp. 178-212.

[3] Warriach, E. U. 2013. State of the art: Embedded middleware platform for a smart home. International Journal of Smart Home, 7(6), pp. 275-294.

[4] Zhang, D., Huang, H., Lai, C. -., Liang, X., Zou, Q., \& Guo, M. 2013. Survey on context-awareness in ubiquitous media. Multimedia Tools and Applications, 67(1), pp. 179-211

[5] Vijay Borges and Wilson Jeberson, 2013. Survey of Context Information Fusion for Sensor Networks based Ubiquitous Systems, J. Sens. Actuator Netw. 2, pp. 1-27

[6] C. Bettini, O. Brdiczka, K. Henricksen, J. Indulska, D. Nicklas, A. Ranganathan, D. Riboni, 2010. A survey of context modelling and reasoning techniques. Pervasive and Mobile Computing, 6 (2), pp. 161-180.

[7] P. Makris, D. Skoutas and C. Skianis 2012. "A survey on context-aware mobile and wireless networking: On networking and computing envi-ronments" integration IEEE Commun. Surveys \& Tutorials, vol. 15, pp. 362386.

[8] Sayed A.Quadri and Othman Sidek Multisensor, 2013. "Data Fusion Algorithm using Factor Analysis Method", International Journal of Advanced Science and Technology, Vol. 55, pp.43-52

[9] Dan C. Marinescu, 2013. Cloud Computing: Theory and Practice, Morgan Kaufmann Publishers In

[10] Xiaoyu Yang, 2013. Principles, Methodologies, and Service-Oriented Approaches for Cloud Computing, Idea Group,U.S.; 1 edition. 